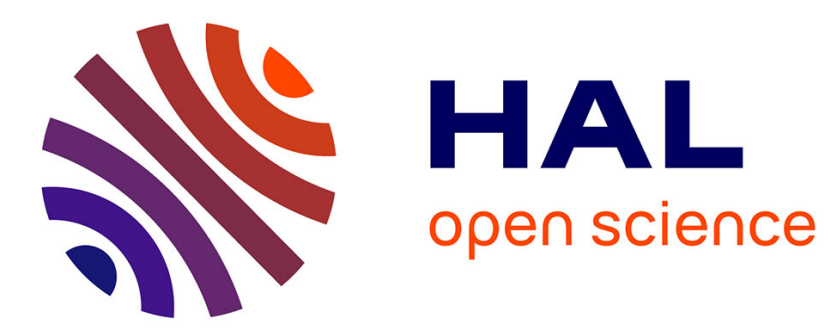

\title{
Reduced-order observer design for descriptor systems with unknown inputs
}

\author{
Mohamed Darouach, Michel Zasadzinski, Mohamed Hayar
}

\section{To cite this version:}

Mohamed Darouach, Michel Zasadzinski, Mohamed Hayar. Reduced-order observer design for descriptor systems with unknown inputs. IEEE Transactions on Automatic Control, 1996, 41 (7), pp.1068-1072. hal-00098122

\section{HAL Id: hal-00098122 \\ https://hal.science/hal-00098122}

Submitted on 24 Sep 2006

HAL is a multi-disciplinary open access archive for the deposit and dissemination of scientific research documents, whether they are published or not. The documents may come from teaching and research institutions in France or abroad, or from public or private research centers.
L'archive ouverte pluridisciplinaire HAL, est destinée au dépôt et à la diffusion de documents scientifiques de niveau recherche, publiés ou non, émanant des établissements d'enseignement et de recherche français ou étrangers, des laboratoires publics ou privés. 


\title{
Reduced-order observer design for descriptor systems with unknown inputs
}

\author{
M. Darouach, M. Zasadzinski and M. Hayar \\ CRAN - CNRS URA 821 - Université de Nancy I \\ 186, rue de Lorraine, Cosnes et Romain, 54400 FRANCE
}

Abstract : A new method for the design of reduced-order observers for descriptor systems with unknown inputs is presented. The approach is based on the generalized constrained Sylvester equation. Sufficient conditions for the existence of the observer are given.

\section{Introduction}

The problem of observers design for standard systems with unknown inputs has received considerable attention in the last two decades ([1]-[4] and references therein). This problem is of great importance in theory and practice since there are many situations where disturbances or partial inputs are inaccessible. In [5] a technique for computing an efficient solution for the unknown input observer design is given. This solution uses the constrained Sylvester equation. The usage of constrained and coupled Sylvester equation in automatic control is well-known [6], [7], and [8]. Recently, a great deal of work has been devoted to the observer design for descriptor systems and many approaches to design such observers exist [9]-[16]. In [9] a method based on the singular value decomposition and the concept of matrix generalized inverse to design a reduced-order observer has been proposed. In [11] the generalized Sylvester equation was used to develop a procedure for designing reduced-order observers. In [12] a method based on the generalized inverse was presented. Observers for continuous descriptor under less restrictive conditions and using only a straightforward matrix manipulation have been presented in [17], [18]. Observers for discrete-time descriptor systems have been developed in [14], [16].

However, only few results have been presented to design observers for descriptor systems with unknown inputs [19], [20], and [21]. Descriptor systems are very sensitive to slight input changes, and the presence of unmeasurable disturbances or unknown inputs is very detrimental to the design of observers. This fact justifies the importance of the observers design for descriptor systems in presence of unknown inputs. On the other hand, many practical systems can be described by descriptor models, and the fault diagnosis of these systems may be based on the unknown input observer design.

In [19] and [20] only square singular systems have been considered under the regularity condition. In addition, the strong observability [19] and the modal observability [20] have been assumed. In [21], a coordinate transformation is used to design a reduced-order observer.

In this paper, we present a new method to design a reduced-order observer for continuoustime descriptor systems subject to unknown inputs and unknown measurement disturbances. As in [21] systems considered are in a general form and less restrictive conditions are required. 


\section{Statement of the problem}

Consider the linear time-invariant descriptor system

$$
\begin{aligned}
& \mathrm{E}^{*} \dot{\mathrm{x}}=\mathrm{A}^{*} \mathrm{x}+\mathrm{B}^{*} \mathrm{u}+\mathrm{F}^{*} \mathrm{w} \\
& \mathrm{y}^{*}=\mathrm{C}^{*} \mathrm{x}+\mathrm{G}^{*} \mathrm{w}
\end{aligned}
$$

where $\mathrm{x} \in \mathbb{R}^{\mathrm{n}}, \mathrm{u} \in \mathbb{R}^{\mathrm{k}}, \mathrm{w} \in \mathbb{R}^{\mathrm{q}}$ and $\mathrm{y}^{*} \in \mathbb{R}^{\mathrm{p}}$ are the state vector, the control input vector, the unmeasurable input vector and the output vector respectively. $\mathrm{E}^{*} \in \mathbb{R}^{\mathrm{mxn}}, \mathrm{A}^{*} \in \mathbb{R}^{\mathrm{mxn}}, \mathrm{B}^{*} \in$ $\mathbb{R}^{\mathrm{mxk}}, \mathrm{F}^{*} \in \mathbb{R}^{\mathrm{mxq}}, \mathrm{G}^{*} \in \mathbb{R}^{\mathrm{pxq}}$, and $\mathrm{C}^{*} \in \mathbb{R}^{\text {pxn }}$ are known constant matrices. We assume that $\operatorname{rank} \mathrm{E}^{*}=\mathrm{r}<\mathrm{n}$, and without loss of generality $\operatorname{rank}\left[\mathrm{C}^{*} \mathrm{G}^{*}\right]=\mathrm{p}$.

Assumptions. In the sequel we assume that

$$
\begin{aligned}
& \text { i) } \operatorname{rank}\left[\begin{array}{c}
\mathrm{F}^{*} \\
\mathrm{G}^{*}
\end{array}\right]=\mathrm{q} \leq \mathrm{p} \\
& \text { ii) } \operatorname{rank}\left[\begin{array}{cccc}
\mathrm{E}^{*} & \mathrm{~A}^{*} & \mathrm{~F}^{*} & 0 \\
0 & \mathrm{E}^{*} & 0 & \mathrm{~F}^{*} \\
0 & \mathrm{C}^{*} & \mathrm{G}^{*} & 0 \\
0 & 0 & 0 & \mathrm{G}^{*}
\end{array}\right]-\operatorname{rank}\left[\begin{array}{cc}
\mathrm{E}^{*} & \mathrm{~F}^{*} \\
0 & \mathrm{G}^{*}
\end{array}\right]=\mathrm{n}+\mathrm{q}
\end{aligned}
$$

These conditions are not restrictive. Condition i) can always be met by redefining the unknown input. If $\operatorname{rank}\left[\begin{array}{l}\mathrm{F}^{*} \\ \mathrm{G}^{*}\end{array}\right]=\mathrm{s}<\mathrm{q}$, then we have $\left[\begin{array}{l}\mathrm{F}^{*} \\ \mathrm{G}^{*}\end{array}\right] \mathrm{w}=\left[\begin{array}{l}\mathrm{F}_{1} * \\ \mathrm{G}_{1} *\end{array}\right] \mathrm{v}$, where $\left[\begin{array}{l}\mathrm{F}_{1} * \\ \mathrm{G}_{1} *\end{array}\right]$ is of full column rank, and v can be considered as a new unknown input. Condition ii) generalizes the condition of the impulse observability of singular square systems (i.e. $m=n$ and det $E^{*}=0$ ) when $\mathrm{F}^{*}=\mathrm{G}^{*}=0$.

For $\mathrm{m}=\mathrm{n}, \mathrm{E}^{*}=\mathrm{I}$, and $\mathrm{G}^{*}=0$, system $(1)$ becomes a standard one with unknown inputs, in this case condition ii) can be written as

$$
\operatorname{rank}\left[\begin{array}{cc}
\mathrm{I} & \mathrm{F}^{*} \\
\mathrm{C}^{*} & 0
\end{array}\right]=\mathrm{n}+\mathrm{q} \text { or equivalently } \operatorname{rank} \mathrm{C}^{*} \mathrm{~F}^{*}=\operatorname{rank} \mathrm{F}^{*}=\mathrm{q}
$$

which is the condition generally assumed in the standard observer for unknown input systems [1]-[4].

Now, since rank $\mathrm{E}^{*}=\mathrm{r}$, there exists a non-singular matrix $\mathrm{P}$ such that

$$
\mathrm{P} \mathrm{E}^{*}=\left[\begin{array}{l}
\mathrm{E} \\
0
\end{array}\right], \mathrm{P} \mathrm{A}^{*}=\left[\begin{array}{c}
\mathrm{A} \\
\mathrm{A}_{1}
\end{array}\right], \mathrm{P} \mathrm{B}^{*}=\left[\begin{array}{c}
\mathrm{B} \\
\mathrm{B}_{1}
\end{array}\right] \text { and } \mathrm{P} \mathrm{F}^{*}=\left[\begin{array}{c}
\mathrm{F} \\
\mathrm{F}_{1}
\end{array}\right]
$$

with $E \in \mathbb{R}^{\text {r.n }}$ and rank $E=r$. Then system (1) is restricted system equivalent (r.s.e) to

$$
\begin{aligned}
& \mathrm{E} \dot{\mathrm{x}}=\mathrm{Ax}+\mathrm{Bu}+\mathrm{Fw} \\
& \mathrm{y}=\mathrm{Cx}+\mathrm{Dw}
\end{aligned}
$$

where $\mathrm{y}=\left[\begin{array}{c}-\mathrm{B}_{1} \mathrm{u} \\ \mathrm{y}^{*}\end{array}\right] \in \mathbb{R}^{\mathrm{t}}, \mathrm{C}=\left[\begin{array}{c}\mathrm{A}_{1} \\ \mathrm{C}^{*}\end{array}\right] \in \mathbb{R}^{\mathrm{t} . \mathrm{n}}$ and $\mathrm{D}=\left[\begin{array}{c}\mathrm{F}_{1} \\ \mathrm{G}^{*}\end{array}\right] \in \mathbb{R}^{\mathrm{t} \cdot \mathrm{q}}$, with $\mathrm{t}=\mathrm{m}+\mathrm{p}-\mathrm{r}$.

One can easily prove that assumption i) is equivalent to rank $\left[\begin{array}{l}F \\ D\end{array}\right]=q$. 
Let rank $\mathrm{D}=\mathrm{q}_{1} \leq \mathrm{q}$, then there exist two non-singular matrices $\mathrm{U}$ and $\mathrm{V}$ such that

$$
\mathrm{UDV}=\left[\begin{array}{cc}
\mathrm{I}_{\mathrm{q}_{1}} & 0 \\
0 & 0
\end{array}\right]
$$

System (2) can now be written as

$$
\begin{aligned}
& \mathrm{E} \dot{\mathrm{x}}=\Phi \mathrm{x}+\mathrm{Bu}+\mathrm{F}_{11} \mathrm{y}_{1}+\mathrm{F}_{12} \mathrm{w}_{2} \\
& \mathrm{y}_{1}=\mathrm{C}_{11} \mathrm{x}+\mathrm{w}_{1}
\end{aligned}
$$

$\begin{aligned} \mathrm{y}_{2} & =\mathrm{C}_{12} \mathrm{x} \\ \text { where }\left[\begin{array}{l}\mathrm{y}_{1} \\ \mathrm{y}_{2}\end{array}\right] & =\mathrm{Uy},\left[\begin{array}{l}\mathrm{C}_{11} \\ \mathrm{C}_{12}\end{array}\right]=\mathrm{UC}, \mathrm{w}=\mathrm{V}\left[\begin{array}{l}\mathrm{w}_{1} \\ \mathrm{w}_{2}\end{array}\right], \mathrm{F} \mathrm{V}=\left[\begin{array}{ll}\mathrm{F}_{11} & \mathrm{~F}_{12}\end{array}\right] \text {, and } \Phi=\mathrm{A}-\mathrm{F}_{11} \mathrm{C}_{11} \text {. }\end{aligned}$

One can easily obtain rank $\mathrm{C}_{12}=\mathrm{p}_{1}=\mathrm{t}-\mathrm{q}_{1}$ from $\operatorname{rank}\left[\begin{array}{cc}C^{*} & \mathrm{G}^{*}\end{array}\right]=\mathrm{p}$ and the above matrix decomposition.

Our aim is to design an observer in the form

$$
\begin{aligned}
& \dot{\mathrm{z}}=\Pi \mathrm{z}+\mathrm{L}_{1} \mathrm{y}_{1}+\mathrm{L}_{2} \mathrm{y}_{2}+\mathrm{H} \mathrm{u} \\
& \hat{\mathrm{x}}=\mathrm{Mz}+\mathrm{N}_{2}
\end{aligned}
$$

where $\mathrm{z} \in \mathbb{R}^{\mathrm{n}-\mathrm{p}_{1}}$.

The problem of the observer design is reduced to finding matrices $\Pi, L_{1}, L_{2}, H, M$ and $N$ such that the estimate $\hat{x}$ converge asymptotically to the state $\mathrm{x}$.

\section{Reduced-order observer design}

In this section we present a new method to design a reduced-order observer for singular system (1) with unknown inputs. The solution of this problem is given in the following theorem.

Theorem 3.1. Let $T$ be an $\left(n-p_{1}\right)$.r matrix such that

$$
\begin{aligned}
& \mathrm{T} \Phi-\Pi \mathrm{T} \mathrm{E}=\mathrm{L}_{2} \mathrm{C}_{12} \\
& \mathrm{~T}_{12}=0
\end{aligned}
$$

where det $\left[\begin{array}{c}\mathrm{TE} \\ \mathrm{C}_{12}\end{array}\right] \neq 0$

Then for

$$
\begin{aligned}
& \mathrm{H}=\mathrm{T} \mathrm{B} \\
& \mathrm{L}_{1}=\mathrm{T} \mathrm{F}_{11}
\end{aligned}
$$

and

$$
\left[\begin{array}{c}
\mathrm{TE} \\
\mathrm{C}_{12}
\end{array}\right]\left[\begin{array}{ll}
\mathrm{M} & \mathrm{N}
\end{array}\right]=\left[\begin{array}{cc}
\mathrm{I}_{\mathrm{n}-\mathrm{p}_{1}} & 0 \\
0 & \mathrm{I}_{\mathrm{p}_{1}}
\end{array}\right]
$$

we have

$$
\hat{x}(t)-x(t)=M e^{\Pi t}(z(0)-\operatorname{TE} x(0))
$$

The convergence of the reduced-order observer is obtained when $\Pi$ is a stability matrix.

Since this theorem is easy to prove, its proof is omitted. 
Equation (5.a) is the so called generalized Sylvester equation, which must be satisfied under the constraint (5.b).

Define the following non-singular matrix

$$
\left[\begin{array}{c}
\mathrm{R} \\
\mathrm{C}_{12}
\end{array}\right]=\left[\begin{array}{cc}
\mathrm{I}_{\mathrm{n}-\mathrm{p}_{1}} & \mathrm{~K} \\
0 & \mathrm{I}_{\mathrm{p}_{1}}
\end{array}\right]\left[\begin{array}{c}
\mathrm{TE} \\
\mathrm{C}_{12}
\end{array}\right]
$$

where $\mathrm{K}$ is an $\left(n-\mathrm{p}_{1}\right) \cdot \mathrm{p}_{1}$ arbitrary matrix and $\mathrm{R}$ is an $\left(n-\mathrm{p}_{1}\right) \cdot \mathrm{n}$ matrix of full row rank, then we have

$$
\mathrm{T} \mathrm{E}=\mathrm{R}-\mathrm{K} \mathrm{C}_{12}
$$

Equations (5.b) and (7) can be written as

$$
[\mathrm{T} \quad \mathrm{K}]\left[\begin{array}{cc}
\mathrm{E} & \mathrm{F}_{12} \\
\mathrm{C}_{12} & 0
\end{array}\right]=\left[\begin{array}{ll}
\mathrm{R} & 0
\end{array}\right]
$$

The solution of this equation depends on the rank of matrix $\left[\begin{array}{cc}E & F_{12} \\ C_{12} & 0\end{array}\right]$. The solution exists if

$$
\operatorname{rank}\left[\begin{array}{cc}
\mathrm{E} & \mathrm{F}_{12} \\
\mathrm{C}_{12} & 0
\end{array}\right]=\mathrm{n}+\operatorname{dim}_{2}
$$

We can now establish the following result.

Lemma 3.1. For systems (1) and (3) we have the following statements:
1) $\operatorname{rank}\left[\begin{array}{cc}E & F_{12} \\ C_{12} & 0\end{array}\right]=n+\operatorname{dim} w_{2}$ if and only if assumption ii) is satisfied.
2) $\operatorname{rank}\left[\begin{array}{cc}\mathrm{sE}-\Phi & -\mathrm{F}_{12} \\ \mathrm{C}_{12} & 0\end{array}\right]=\operatorname{rank}\left[\begin{array}{cc}\mathrm{sE}^{*}-\mathrm{A}^{*} & -\mathrm{F}^{*} \\ \mathrm{C}^{*} & \mathrm{G}^{*}\end{array}\right]-\mathrm{q}_{1}, \forall \mathrm{s} \in \mathbb{C}$.

Proof. 1) Define the following non-singular matrices

$$
\begin{aligned}
& \mathrm{U}_{1}=\left[\begin{array}{llll}
\mathrm{P} & 0 & 0 & 0 \\
0 & \mathrm{P} & 0 & 0 \\
0 & 0 & \mathrm{I}_{\mathrm{p}} & 0 \\
0 & 0 & 0 & \mathrm{I}_{\mathrm{p}}
\end{array}\right], \mathrm{V}_{1}=\left[\begin{array}{llll}
\mathrm{Q} & 0 & 0 & 0 \\
0 & \mathrm{Q} & 0 & 0 \\
0 & 0 & \mathrm{I}_{\mathrm{q}} & 0 \\
0 & 0 & 0 & \mathrm{I}_{\mathrm{q}}
\end{array}\right], \mathrm{U}_{2}=\left[\begin{array}{ll}
\mathrm{P} & 0 \\
0 & \mathrm{I}_{\mathrm{p}}
\end{array}\right], \mathrm{V}_{2}=\left[\begin{array}{ll}
\mathrm{Q} & 0 \\
0 & \mathrm{I}_{\mathrm{q}}
\end{array}\right], \\
& \mathrm{U}_{3}=\left[\begin{array}{lll}
\mathrm{U} & 0 & 0 \\
0 & \mathrm{I}_{\mathrm{r}} & 0 \\
0 & 0 & \mathrm{U}
\end{array}\right], \mathrm{V}_{3}=\left[\begin{array}{ccc}
\mathrm{I}_{\mathrm{n}} & 0 & 0 \\
0 & \mathrm{~V} & 0 \\
0 & 0 & \mathrm{~V}
\end{array}\right], \mathrm{U}_{4}=\left[\begin{array}{ll}
\mathrm{I}_{\mathrm{r}} & 0 \\
0 & \mathrm{U}
\end{array}\right], \mathrm{V}_{4}=\left[\begin{array}{cc}
\mathrm{I}_{\mathrm{n}} & 0 \\
0 & \mathrm{~V}
\end{array}\right] \text { and }
\end{aligned}
$$




$$
\mathrm{U}_{5}=\left[\begin{array}{ccc}
\mathrm{I}_{\mathrm{r}} & \mathrm{F}_{11} & 0 \\
0 & \mathrm{I}_{\mathrm{q}_{1}} & 0 \\
0 & 0 & \mathrm{I}_{\mathrm{p}_{1}}
\end{array}\right]
$$

then from assumption ii) we obtain

$$
\operatorname{rank} U_{1}\left[\begin{array}{cccc}
\mathrm{E}^{*} & \mathrm{~A}^{*} & \mathrm{~F}^{*} & 0 \\
0 & \mathrm{E}^{*} & 0 & \mathrm{~F}^{*} \\
0 & \mathrm{C}^{*} & \mathrm{G}^{*} & 0 \\
0 & 0 & 0 & \mathrm{G}^{*}
\end{array}\right] \mathrm{V}_{1}-\operatorname{rank} \mathrm{U}_{2}\left[\begin{array}{cc}
\mathrm{E}^{*} & \mathrm{~F}^{*} \\
0 & \mathrm{G}^{*}
\end{array}\right] \mathrm{V}_{2}=\mathrm{n}+\mathrm{q}
$$

or equivalently rank $\left[\begin{array}{ccc}C & D & 0 \\ E & 0 & F \\ 0 & 0 & D\end{array}\right]-\operatorname{rank} D=n+q$, since $E$ is of full row rank.

Then rank $U_{3}\left[\begin{array}{ccc}C & D & 0 \\ E & 0 & F \\ 0 & 0 & D\end{array}\right] V_{3}-\operatorname{rank} U D V=n+q$, it follows that

$$
\operatorname{rank}\left[\begin{array}{cc}
\mathrm{E} & \mathrm{F}_{12} \\
\mathrm{C}_{12} & 0
\end{array}\right]=\mathrm{n}+\operatorname{dim} \mathrm{w}_{2}
$$

2) We have the following relations

$$
\begin{aligned}
\operatorname{rank}\left[\begin{array}{cc}
\mathrm{sE}^{*}-\mathrm{A}^{*} & -\mathrm{F}^{*} \\
\mathrm{C}^{*} & \mathrm{G}^{*}
\end{array}\right] & =\operatorname{rank} \mathrm{U}_{2}\left[\begin{array}{cc}
\mathrm{sE}^{*}-\mathrm{A}^{*} & -\mathrm{F}^{*} \\
\mathrm{C}^{*} & \mathrm{G}^{*}
\end{array}\right] \mathrm{V}_{2} \\
& =\operatorname{rank} \mathrm{U}_{4}\left[\begin{array}{cc}
\mathrm{sE}-\mathrm{A} & -\mathrm{F} \\
\mathrm{C} & \mathrm{D}
\end{array}\right] \mathrm{V}_{4} \\
& =\operatorname{rank} \mathrm{U}_{5}\left[\begin{array}{ccc}
\mathrm{sE}-\Phi & -\mathrm{F}_{11} & -\mathrm{F}_{12} \\
\mathrm{C}_{11} & \mathrm{I}_{\mathrm{q}_{1}} & 0 \\
\mathrm{C}_{12} & 0 & 0
\end{array}\right] \\
& =\operatorname{rank}\left[\begin{array}{cc}
\mathrm{sE}-\Phi & -\mathrm{F}_{12} \\
\mathrm{C}_{12} & 0
\end{array}\right]+\mathrm{q}_{1}
\end{aligned}
$$

Now, equation (7) can be written as

$$
\left[\begin{array}{ll}
\mathrm{T} & \mathrm{K}
\end{array}\right]\left[\begin{array}{c}
\mathrm{E} \\
\mathrm{C}_{12}
\end{array}\right]=\mathrm{R}
$$

It follows from (9) that rank $\left[\begin{array}{c}E \\ C_{12}\end{array}\right]=n$, then the general solution of (11) is therefore given by 


$$
\left[\begin{array}{ll}
\mathrm{T} & \mathrm{K}
\end{array}\right]=\mathrm{R}\left[\begin{array}{c}
\mathrm{E} \\
\mathrm{C}_{12}
\end{array}\right]+\mathrm{Y}\left(\mathrm{I}_{\mathrm{r}+\mathrm{p}_{1}}-\left[\begin{array}{c}
\mathrm{E} \\
\mathrm{C}_{12}
\end{array}\right] \cdot\left[\begin{array}{c}
\mathrm{E} \\
\mathrm{C}_{12}
\end{array}\right]+\right)
$$

where $\left[\begin{array}{c}E \\ C_{12}\end{array}\right]+$ is the generalized inverse of matrix $\left[\begin{array}{c}E \\ C_{12}\end{array}\right]$, given by $\left[\begin{array}{c}E \\ C_{12}\end{array}\right]+=\Delta\left[E^{T} C_{12}^{T}\right]$, where $\Delta=\left(\mathrm{E}^{\mathrm{T}} \mathrm{E}+\mathrm{C}_{12}{ }^{\mathrm{T}} \mathrm{C}_{12}\right)^{-1}$ and $\mathrm{Y}$ is an arbitrary matrix of appropriate dimension.

From equation (12), we have

$$
\mathrm{T}=\mathrm{R} \Delta \mathrm{E}^{\mathrm{T}}+\mathrm{Y} \varphi
$$

where $\varphi=\left[\begin{array}{c}I_{r}-E \Delta E^{T} \\ -C_{12} \Delta E^{T}\end{array}\right]$. Substituting (13) into (5.b) gives

$$
\mathrm{Y} \varphi \mathrm{F}_{12}=-\mathrm{R} \Delta \mathrm{E}^{\mathrm{T}} \mathrm{F}_{12}
$$

The solution of this equation exists if $\varphi \mathrm{F}_{12}$ is of full column rank. We now state the following result.

Lemma 3.2. The matrix $\left(\varphi F_{12}\right)$ is of full column rank if and only if assumption ii) is satisfied.

Proof. Define the following full column rank matrix

$$
S=\left[\begin{array}{c}
{\left[\begin{array}{c}
E \\
C_{12}
\end{array}\right]+} \\
\left(I_{r+p_{1}}-\left[\begin{array}{c}
E \\
C_{12}
\end{array}\right] \cdot\left[\begin{array}{c}
E \\
C_{12}
\end{array}\right]+\right)
\end{array}\right],
$$

then from lemma 3.1 we have

$$
\begin{aligned}
\operatorname{rank}\left[\begin{array}{cc}
\mathrm{E} & \mathrm{F}_{12} \\
\mathrm{C}_{12} & 0
\end{array}\right] & =\mathrm{n}+\operatorname{dim} \mathrm{w}_{2} \\
& =\operatorname{rank} \mathrm{S}\left[\begin{array}{cc}
\mathrm{E} & \mathrm{F}_{12} \\
\mathrm{C}_{12} & 0
\end{array}\right]
\end{aligned}
$$

it follows that rank $\varphi \mathrm{F}_{12}=\operatorname{dim} \mathrm{w}_{2}$ if and only if assumption ii) is verified.

From lemma 3.2 the solution of (14) is given by

$$
\mathrm{Y}=-\mathrm{R} \Delta \mathrm{E}^{\mathrm{T}} \mathrm{F}_{12}\left(\varphi \mathrm{F}_{12}\right)^{+}+\mathrm{Z}\left(\mathrm{I}_{\mathrm{r}+\mathrm{p}_{1}}-\left(\varphi \mathrm{F}_{12}\right)\left(\varphi \mathrm{F}_{12}\right)^{+}\right)
$$

substituting (15) into (13) gives 


$$
\mathrm{T}=\mathrm{R} \Delta \mathrm{E}^{\mathrm{T}}-\mathrm{R} \Delta \mathrm{E}^{\mathrm{T}} \mathrm{F}_{12}\left(\varphi \mathrm{F}_{12}\right)^{+} \varphi+\mathrm{Z}\left(\mathrm{I}_{\mathrm{r}+\mathrm{p}_{1}}-\left(\varphi \mathrm{F}_{12}\right)\left(\varphi \mathrm{F}_{12}\right)^{+}\right) \varphi
$$

where $\mathrm{Z}$ is an arbitrary matrix.

Now, from (5.a) and (5.e) we have

$$
\begin{aligned}
& \Pi=T \Phi M \\
& L_{2}=T \Phi N
\end{aligned}
$$

Substituting (16) into (17) gives

$$
\begin{array}{ll} 
& \Pi=\Omega+\mathrm{Z} \Gamma \\
\text { where } \quad & \Omega=\mathrm{R} \Delta \mathrm{E}^{\mathrm{T}} \Phi \mathrm{M}-\mathrm{R} \Delta \mathrm{E}^{\mathrm{T}} \mathrm{F}_{12}\left(\varphi \mathrm{F}_{12}\right)^{+} \varphi \Phi \mathrm{M} \\
\text { and } \Gamma=\left(\mathrm{I}_{\mathrm{r}+\mathrm{p}_{1}}-\left(\varphi \mathrm{F}_{12}\right)\left(\varphi \mathrm{F}_{12}\right)^{+}\right) \varphi \Phi \mathrm{M}
\end{array}
$$

If the pair $(\Omega, \Gamma)$ is detectable, one can design a reduced-order observer from the standard methods, in the form (4).

In the sequel, we will give sufficient conditions for the existence of the reduced-order observer.

Lemma 3.3. Under the assumption rank $\left[\begin{array}{c}E \\ C_{12}\end{array}\right]=n$, the reduced-order observer (4) exists if

$$
\operatorname{rank}\left[\begin{array}{cc}
\mathrm{sE}-\Phi & -\mathrm{F}_{12} \\
\mathrm{C}_{12} & 0
\end{array}\right]=\mathrm{n}+\operatorname{dim} \mathrm{w}_{2}, \forall \mathrm{s} \in \mathbb{C}, \operatorname{Re}(\mathrm{s}) \geq 0
$$

Proof. First note that rank $\left[\begin{array}{cc}\mathrm{sE}-\Phi & -\mathrm{F}_{12} \\ \mathrm{C}_{12} & 0\end{array}\right]=\operatorname{rank}\left[\begin{array}{ccc}\mathrm{I}_{\mathrm{r}} & 0 & 0 \\ 0 & \mathrm{I}_{\mathrm{p}_{1}} & -\mathrm{sI}_{\mathrm{p}_{1}} \\ 0 & 0 & \mathrm{I}_{\mathrm{p}_{1}}\end{array}\right]\left[\begin{array}{cc}\mathrm{sE}-\Phi & -\mathrm{F}_{12} \\ \mathrm{sC}_{12} & 0 \\ \mathrm{C}_{12} & 0\end{array}\right]$

$$
=\operatorname{rank}\left[\begin{array}{cc}
\mathrm{sE}-\Phi-\mathrm{F}_{12} \\
\mathrm{sC}_{12} & 0 \\
\mathrm{C}_{12} & 0
\end{array}\right]
$$

Now, define the following matrices $S_{1}=\left[\begin{array}{cc}{\left[\begin{array}{c}\mathrm{R} \\ \mathrm{C}_{12}\end{array}\right] \cdot\left[\begin{array}{c}\mathrm{E} \\ \mathrm{C}_{12}\end{array}\right]+} & 0 \\ \left(\mathrm{I}_{\mathrm{r}+\mathrm{p}_{1}}-\left[\begin{array}{c}\mathrm{E} \\ \mathrm{C}_{12}\end{array}\right] \cdot\left[\begin{array}{c}\mathrm{E} \\ \mathrm{C}_{12}\end{array}\right]+\right)^{+} & 0 \\ 0 & \mathrm{I}_{\mathrm{p}_{1}}\end{array}\right]$,

$$
\mathrm{S}_{2}=\left[\begin{array}{ccc}
\mathrm{M} & \mathrm{N} & 0 \\
0 & 0 & -\mathrm{I}_{\text {dimw }_{2}}
\end{array}\right] \text {, and } \mathrm{S}_{3}=\left[\begin{array}{ccc}
\mathrm{I}_{\mathrm{n}-\mathrm{p}_{1}} & 0 & -\mathrm{R} \Delta \mathrm{E}^{\mathrm{T}} \mathrm{F}_{12}\left(\varphi \mathrm{F}_{12}\right)^{+} \\
0 & \mathrm{I}_{\mathrm{p}_{1}} & -\mathrm{C}_{12} \Delta \mathrm{E}^{\mathrm{T}} \mathrm{F}_{12}\left(\varphi \mathrm{F}_{12}\right)^{+} \\
0 & 0 & \left(\varphi \mathrm{F}_{12}\right)^{+} \\
0 & 0 & \mathrm{I}_{\mathrm{r}+\mathrm{p}_{1}}-\left(\varphi \mathrm{F}_{12}\right)\left(\varphi \mathrm{F}_{12}\right)^{+}
\end{array}\right] \text {, }
$$


then we have

$$
\begin{aligned}
& \operatorname{rank}\left[\begin{array}{cc}
\mathrm{sE}-\Phi & -\mathrm{F}_{12} \\
\mathrm{sC}_{12} & 0 \\
\mathrm{C}_{12} & 0
\end{array}\right]=\operatorname{rank} \mathrm{S}_{1}\left[\begin{array}{cc}
\mathrm{sE}-\Phi-\mathrm{F}_{12} \\
\mathrm{sC}_{12} & 0 \\
\mathrm{C}_{12} & 0
\end{array}\right] \mathrm{S}_{2} \\
& =\operatorname{rank}\left[\begin{array}{ccc}
\mathrm{sI}_{\mathrm{n}-\mathrm{p}_{1}}-\mathrm{R} \Delta \mathrm{E}^{\mathrm{T}} \Phi \mathrm{M} & \left(\mathrm{sR}-\mathrm{R} \Delta \mathrm{E}^{\mathrm{T}} \Phi\right) \mathrm{N} & \mathrm{R} \Delta \mathrm{E}^{\mathrm{T}} \mathrm{F}_{12} \\
-\mathrm{C}_{12} \Delta \mathrm{E}^{\mathrm{T}} \Phi \mathrm{M} & \left(\mathrm{sC}_{12}-\mathrm{C}_{12} \Delta \mathrm{E}^{\mathrm{T}} \Phi\right) \mathrm{N} & \mathrm{C}_{12} \Delta \mathrm{E}^{\mathrm{T}} \mathrm{F}_{12} \\
-\varphi \Phi \mathrm{M} & -\varphi \Phi \mathrm{N} & \varphi \mathrm{F}_{12} \\
0 & \mathrm{I}_{\mathrm{p}_{1}} & 0
\end{array}\right], \\
& =\mathrm{n}+\operatorname{dim} \mathrm{w}_{2}, \forall \mathrm{s} \in \mathbb{C}, \operatorname{Re}(\mathrm{s}) \geq 0,
\end{aligned}
$$

Since $R M=I_{n-p_{1}}$ and $C_{12} M=0$, then it follows that

$$
\operatorname{rank}\left[\begin{array}{cc}
\mathrm{sI}_{\mathrm{n}-\mathrm{p}_{1}}-\mathrm{R} \Delta \mathrm{E}^{\mathrm{T}} \Phi \mathrm{M} & \mathrm{R} \Delta \mathrm{E}^{\mathrm{T}} \mathrm{F}_{12} \\
-\mathrm{C}_{12} \Delta \mathrm{E}^{\mathrm{T}} \Phi \mathrm{M} & \mathrm{C}_{12} \Delta \mathrm{E}^{\mathrm{T}} \mathrm{F}_{12} \\
-\varphi \Phi \mathrm{M} & \varphi \mathrm{F}_{12}
\end{array}\right]=\operatorname{dim} \mathrm{w}_{2}+\mathrm{n}-\mathrm{p}_{1}, \forall \mathrm{s} \in \mathbb{C}, \operatorname{Re}(\mathrm{s}) \geq 0
$$

or equivalently

$$
\begin{aligned}
& \operatorname{rank} S_{3}\left[\begin{array}{cc}
\mathrm{sI}_{\mathrm{n}-\mathrm{p}_{1}}-\mathrm{R} \Delta \mathrm{E}^{\mathrm{T}} \Phi \mathrm{M} & \mathrm{R} \Delta \mathrm{E}^{\mathrm{T}} \mathrm{F}_{12} \\
-\mathrm{C}_{12} \Delta \mathrm{E}^{\mathrm{T}} \Phi \mathrm{M} & \mathrm{C}_{12} \Delta \mathrm{E}^{\mathrm{T}} \mathrm{F}_{12} \\
-\varphi \Phi \mathrm{M} & \varphi \mathrm{F}_{12}
\end{array}\right] \\
& =\operatorname{rank}\left[\begin{array}{cc}
\mathrm{sI}_{\mathrm{n}-\mathrm{p}_{1}}-\Omega & 0 \\
-\mathrm{C}_{12} \Delta \mathrm{E}^{\mathrm{T}} \Phi \mathrm{M}+\mathrm{C}_{12} \Delta \mathrm{E}^{\mathrm{T}} \mathrm{F}_{12}\left(\varphi \mathrm{F}_{12}\right)^{+} \varphi \Phi \mathrm{M} & 0 \\
\left(\varphi \mathrm{F}_{12}\right)^{+} \varphi \Phi \mathrm{M} & \mathrm{I}_{\mathrm{dimw}_{2}} \\
\Gamma & 0
\end{array}\right]
\end{aligned}
$$$$
=\operatorname{dim} \mathrm{w}_{2}+\mathrm{n}-\mathrm{p}_{1}, \forall \mathrm{s} \in \mathbb{C}, \operatorname{Re}(\mathrm{s}) \geq 0
$$

which gives rank $\left[\begin{array}{cc}\mathrm{sI}_{\mathrm{n}-\mathrm{p}_{1}}-\Omega \\ 0 & \mathrm{I}_{\mathrm{p}_{1}} \\ \Gamma\end{array}\right]=\mathrm{n}-\mathrm{p}_{1}$, since $\mathrm{C}_{12} \Delta \mathrm{E}^{\mathrm{T}}=-\left[\begin{array}{ll}0 & \mathrm{I}_{\mathrm{p}_{1}}\end{array}\right] \varphi$, and finally we have $\operatorname{rank}\left[\begin{array}{c}\mathrm{sI}_{\mathrm{n}-\mathrm{p}_{1}}-\Omega \\ \Gamma\end{array}\right]=\mathrm{n}-\mathrm{p}_{1}, \forall \mathrm{s} \in \mathbb{C}, \operatorname{Re}(\mathrm{s}) \geq 0$

which is the detectability of the pair $(\Omega, \Gamma)$, and $\Pi$ is a stability matrix.

The above results leads to the following theorem.

Theorem 3.2. Sufficient conditions for the observer (4) to exist are assumptions i), ii) and

$$
\operatorname{rank}\left[\begin{array}{cc}
\mathrm{sE}^{*}-\mathrm{A}^{*} & -\mathrm{F}^{*} \\
\mathrm{C}^{*} & \mathrm{G}^{*}
\end{array}\right]=\mathrm{n}+\mathrm{q}, \forall \mathrm{s} \in \mathbb{C}, \operatorname{Re}(\mathrm{s}) \geq 0
$$

The proof can be straightforwardly deduced from lemma 3.1, 3.2 and 3.3, and is omitted. 
Remarks: i) Condition (23) generalizes the notion of stable transmission zeros of the square singular systems.

ii) The minimal order $\rho$ of the observer is given by : $\rho=n-\operatorname{rank} C_{12}$.

iii) Matrix $Z$ in equation (19) can be chosen to make the poles of the observer have specified locations if, in theorem $3.2, \forall \mathrm{s} \in \mathbb{C}, \operatorname{Re}(\mathrm{s}) \geq 0$ is replaced by $\forall \mathrm{s} \in \mathbb{C}$.

iv) The design of the observer presented in this paper requires only straightforward matrix operations. A procedure to compute efficiently the observer matrices can be obtained from the singular values decomposition of the full column matrices $\left(\varphi F_{12}\right)$ and $\left[\begin{array}{c}E \\ C_{12}\end{array}\right]$. This problem has not been presented in this paper, the reader can refer to [8] for more details on the computations problems.

Now, we can summarize the procedure for designing the observer :

1) Choose an (n- $\left.p_{1}\right) \cdot n$ matrix $R$ such that $\left[\begin{array}{c}R \\ C_{12}\end{array}\right]$ is non-singular, this can be always done, since $\mathrm{C}_{12}$ is of full row rank.

2) The matrix $M$ can be obtained from (5.e) and (11)

$$
M=\left[\begin{array}{c}
R \\
C_{12}
\end{array}\right]^{-1}\left[\begin{array}{c}
I_{n}-p_{1} \\
0
\end{array}\right]
$$

3) Compute

$$
\alpha=\mathrm{I}_{\mathrm{r}+\mathrm{p}_{1}}-\mathrm{F}_{12}\left(\varphi \mathrm{F}_{12}\right)^{+} \varphi, \Delta=\left(\left[\begin{array}{c}
\mathrm{E} \\
\mathrm{C}_{12}
\end{array}\right] \mathrm{T}\left[\begin{array}{c}
\mathrm{E} \\
\mathrm{C}_{12}
\end{array}\right]\right)^{-1}, \Omega=\mathrm{R} \Delta \mathrm{E}^{\mathrm{T}} \alpha \Phi \mathrm{M},
$$

and $\Gamma=\varphi \alpha \Phi \mathrm{M}$.

4) If the pair $(\Omega, \Gamma)$ is detectable, we can find $\mathrm{Z}$ such that the observer is asymptotically stable, then we can compute $\mathrm{T}=\left(\mathrm{R} \Delta \mathrm{E}^{\mathrm{T}}+\mathrm{Z} \varphi\right) \alpha$.

5) The matrix $\mathrm{N}$ can be obtained from (5.e)

$$
\mathrm{N}=\left[\begin{array}{c}
\mathrm{TE} \\
\mathrm{C}_{12}
\end{array}\right]^{-1}\left[\begin{array}{c}
0 \\
\mathrm{I}_{\mathrm{p}_{1}}
\end{array}\right]
$$

6) Matrices $\mathrm{H}, \mathrm{L}_{1}$ and $\mathrm{L}_{2}$ can be obtained from (5.c), (5.d) and (18).

\section{Numerical example}

The following example illustrates the above design method. Consider the singular system (1) described by [19] 


$$
\begin{aligned}
& \mathrm{E}^{*}=\left[\begin{array}{llll}
1 & 0 & 0 & 0 \\
0 & 1 & 0 & 0 \\
0 & 0 & 1 & 0 \\
0 & 0 & 0 & 0
\end{array}\right], \mathrm{A}^{*}=\left[\begin{array}{cccc}
-1 & 1 & 0 & 0 \\
-1 & 0 & 0 & 1 \\
0 & -1 & -1 & 0 \\
0 & 0 & 0 & 1
\end{array}\right], \mathrm{B}^{*}=\left[\begin{array}{ll}
1 & 0 \\
0 & 1 \\
0 & 0 \\
1 & 0
\end{array}\right], \mathrm{F}^{*}=\left[\begin{array}{c}
-1 \\
0 \\
0 \\
0
\end{array}\right], \mathrm{G}^{*}=\left[\begin{array}{l}
0 \\
0
\end{array}\right] \\
& \text { and } \mathrm{C}^{*}=\left[\begin{array}{llll}
1 & 0 & 0 & 0 \\
0 & 0 & 1 & 1
\end{array}\right]
\end{aligned}
$$

In this case the transformation matrix is $\mathrm{P}=\mathrm{I}_{4}$. We obtain the r.s.e singular system (4) described by

$$
\begin{aligned}
& \mathrm{E}=\left[\begin{array}{llll}
1 & 0 & 0 & 0 \\
0 & 1 & 0 & 0 \\
0 & 0 & 1 & 0
\end{array}\right], \mathrm{A}=\left[\begin{array}{cccc}
-1 & 1 & 0 & 0 \\
-1 & 0 & 0 & 1 \\
0 & -1 & -1 & 0
\end{array}\right], \mathrm{B}=\left[\begin{array}{ll}
1 & 0 \\
0 & 1 \\
0 & 0
\end{array}\right], \mathrm{F}=\left[\begin{array}{c}
-1 \\
0 \\
0
\end{array}\right], \mathrm{D}=\left[\begin{array}{l}
0 \\
0 \\
0
\end{array}\right], \\
& \mathrm{C}=\left[\begin{array}{llll}
1 & 0 & 0 & 0 \\
0 & 0 & 1 & 1
\end{array}\right] \text { and } \mathrm{y}=\left[\begin{array}{l}
-\mathrm{u}_{1} \\
\mathrm{y}^{*}
\end{array}\right]
\end{aligned}
$$

Choose $\mathrm{R}=\left[\begin{array}{llll}0 & 1 & 0 & 0\end{array}\right]$, then

$$
M=\left[\begin{array}{l}
0 \\
1 \\
0 \\
0
\end{array}\right], \Omega=0 \text { and } \Gamma=\left[\begin{array}{c}
\gamma \\
0 \\
-1 / 3 \\
-1 / 3 \\
-\gamma \\
1 / 3
\end{array}\right] \text { where } \gamma \in R
$$

For $\Pi=-1$, we can choose $\mathrm{Z}=\left[\begin{array}{llllll}0 & 0 & 3 & 3 & 0 & 3\end{array}\right]$, then

$$
\mathrm{T}=\left[\begin{array}{lll}
0 & 1 & 1
\end{array}\right], \mathrm{N}=\left[\begin{array}{ccc}
0 & 1 & 0 \\
1 & 0 & -1 \\
-1 & 0 & 1 \\
1 & 0 & 0
\end{array}\right], \mathrm{L}_{1}=0, \mathrm{~L}_{2}=\left[\begin{array}{lll}
1 & -1 & 0
\end{array}\right] \text { and } \mathrm{H}=\left[\begin{array}{ll}
0 & 1
\end{array}\right]
$$

Thus, the reduced-order observer is

$$
\begin{gathered}
\dot{\mathrm{z}}=-\mathrm{z}+\mathrm{y}_{1}-\mathrm{y}_{2}+\mathrm{u}_{2} \\
\hat{\mathrm{x}}=\left[\begin{array}{c}
\mathrm{y}_{2} \\
\mathrm{z}+\mathrm{y}_{1}-\mathrm{y}_{3} \\
-\mathrm{y}_{1}+\mathrm{y}_{3} \\
\mathrm{y}_{1}
\end{array}\right]
\end{gathered}
$$

\section{Conclusion}

We have presented a systematic design method for a reduced-order observer for linear singular systems with unknown inputs. This method is based on the constrained generalized 
Sylvester equation. The existence conditions of the observer are given and generalize those currently used for unknown inputs observer problem of singular systems.

\section{References}

[1] F. Yang and R.W. Wilde, "Observers for linear systems with unknown inputs", IEEE Trans. Automat. Control, vol 33, pp 677-681, 1988.

[2] Y.P. Guan and M. Saif, "A novel approach to the design of unknown input observers", IEEE Trans. Automat. Control, vol 36, pp 632-635, 1991.

[3] M. Hou and P.C. Müller, "Design of observers for linear systems with unknown inputs", IEEE Trans. Automat. Control, vol 37, pp 871-875, 1992.

[4] M. Darouach, M. Zasadzinski and S.J. Xu, "Full-order observers for linear systems with unknown inputs", IEEE Trans. Automat. Control, vol 39, pp 606-609, 1994.

[5] V.L. Syrmos, "Computational observer design techniques for linear systems with unknown inputs using the concept of transmission zeros", IEEE Trans. Automat. Control, vol 38, pp 790-794, 1993.

[6] V.L. Syrmos, "Disturbance decoupling using constrained Sylvester equations", IEEE Trans. Automat. Control, vol 39, pp 797-803, 1994.

[7] V.L. Syrmos and F.L. Lewis, "Constrained and coupled Sylvester equations in systems design”, CSSP Special Issue, Eds. A.J. Laub, V.L. Syrmos and F.L. Lewis, vol 13, pp 663-694, 1994.

[8] R.V. Patel, A.J. Laub and P.M. Van Dooren, Numerical Linear Algebra Techniques for Systems and Control, IEEE Press, 1994.

[9] M. El-Tohami, V. Lovass-Nagy and R. Mukundan, "On the design of observers for generalized state space systems using singular value decomposition”, Int. J. Control, vol 38, pp 673-683, 1983.

[10] M. El-Tohami, V. Lovass-Nagy, and D.L. Powers, "Input function observers for generalized state space systems", Int. J. Control, vol 40, pp 903-922, 1984.

[11] M. Verhaegen and P.M. Van Dooren, "A reduced observer for descriptor systems", Systems \& Control Letters, vol 8, pp 29-37, 1986.

[12] B. Shafai and R.L. Caroll, "Design of minimal order observers for singular systems", Int. J. Control, vol 45, pp 1075-1081, 1987.

[13] M.M. Fahmy and J. O'Reilly, "Observer for descriptor systems", Int. J. Control, vol 49, pp 2013-2028, 1989.

[14] L. Dai, "Observer for discrete singular systems", IEEE Trans. Automat. Control, vol 33, pp 187-191, 1988.

[15] P.N. Paraskevopoulos and F.N. Kounboulis, "Observers for singular systems", IEEE Trans. Automat. Control, vol 37, pp 1211-1215, 1992.

[16] L. Dai, Singular Control Systems, Springer-Verlag, Berlin, 1989.

[17] M. Darouach and M. Boutayeb, "Observer design for descriptor systems", IEEE Trans. Automat. Control, vol 40, pp 1323 - 1327, 1995

[18] P.C. Müller and M. Hou, "On the observer design for descriptor systems", IEEE Trans. Automat. Control, vol 38, pp 1666-1671, 1993.

[19] C.W. Yang and H.L. Tan, "Observer design for singular systems with unknown inputs", Int. J. Control, vol 49, pp 1937-1946, 1989.

[20] P.N. Paraskevopoulos, F.N. Koumboulis, K.G. Tzierakis and G.E. Panagiotakis, "Observers design for generalized state space systems with unknown inputs", Systems \& Control Letters, vol 18, pp 309-321, 1992.

[21] M. Hou and P.C. Muller, " Observer for linear descriptor systems with unknown inputs" (in German), Automatisierungstechnic, vol 40, pp 220-227, 1992. 